\title{
Eco-Taxonomic Study of Family Poaceae (Gramineae)
}

\author{
Muhammad Nauman Khan ${ }^{1,2, *}$, Sajjad Ali', Tabassum Yaseen¹, Sami Ullah², Akhtar Zaman², Majid Iqbal³, \\ Sikandar Shah ${ }^{2}$ \\ ${ }^{1}$ Department of Botany, Bacha Khan University, Charsadda, KP, Pakistan \\ 2Department of Botany, University of Peshawar, Peshawar, KP, Pakistan \\ 3Plant Ecology and Conservation Laboratory, Department of Plant Sciences, Quaid-e-Azam University, Islamabad, Pakistan
}

\section{ABSTRACT}

Background: The study of species diversity, its conservations and extinction can be done by using systematics in plant biodiversity. Poaceae is a very diverse grass family with great economic importance as it contains crops like rice, maize, oats, wheat, etc.

Objectives: The current study was undertaken in district Charsadda during 2017-18 in flowering season to collect different grass species from the area.

Methodology: Grass specimens were gathered, preserved, mounted and then identified from the available literature/native flora (Flora of Pakistan). Ecological parameters like habitat, class, life cycle and biological spectra were determined. A total of 51 taxa comprised of 34 genera, 5 subfamilies and 11 tribes of grasses were collected from the District Charsadda. The subfamily Panicoideae was the leading family with highest number of genera, taxa and tribes, followed by Pooideae.

Results: The biological spectrum showed that in the life-form class, Therophyte had the highest number of species $32(62.74 \%)$, followed by Hemicryptophyte having 13 species $(25.49 \%)$. In the Leaf size class, microphylls were dominant with 24 species $(47.05 \%)$ followed by nanophylls having 14 species $(27.45 \%)$. The adaptational survival of plants showed that 32 species $(62.74 \%)$ were terrestrial and 19 species (37.25\%) were amphibious in our study. The Life-cycle class depicted that 33 species (64.70\%) were annual, 17 species $(33.33 \%)$ were perennial and 1 species $(1.960 \%)$ was annual or perennial. The Palatability class showed that 35 species $(68.62 \%)$ were highly palatable, followed by less-palatable 11 species $(21.56 \%)$, non-palatable 2 species $(3.921 \%)$ and moderately palatable 3 species (5.882\%). The Abundant class were determined through species rating scale in ecology ACFOR (Abundant Common Frequent Occasional Rare) scale to describe species abundance in a given area; 26 species (50.98\%) were occasional, 12 species (23.52\%) were frequent, 7 species (13.72\%) were rare, 5 species $(9.803 \%)$ were common and only 1 species Cynodon dactylon (1.960\%) was abundant in the whole area and present everywhere in the research area in every season.

Conclusion: It has been concluded from the study that unwise collection, over-grazing, over-exploitation, over-consumption and overutilization are major biotic factors, which affect the diversity of the grasses in the area and affect the populations' sustainability on the earth crust. Therefore, the study aims to document and explore wild grasses from the area, which might help in future researches. This survey will be helpful in identifying plant wealth and status for their exploitation on systematic approaches and scientific basis.

$\begin{array}{lll}\text { Keywords } & \text { *Address of Correspondence } & \text { Article info. } \\ \text { Grasses, Systematic, Biological Spectra, } & \text { khannauman41@gmail.com } & \text { Received: January 29, } 2019 \\ \text { Tribes, Abundant Class, Charsadda. } & & \text { Accepted: October 25, 2019 }\end{array}$

Cite this article: Khan MN, Ali S, Yaseen T, Ullah S, Zaman A, Iqbal M, et al. Eco-Taxonomic

Study of Family Poaceae (Gramineae). RADS J Biol Res Appl Sci. 2019; 10(2):63-75.

This is an Open Access article distributed under the terms of the Creative Commons Attribution License

Funding Source: Nil

Conflict of Interest: Nil

(http://creativecommons.org/licenses/by/4.0), which permits unrestricted use, distribution, and reproduction in

any medium, provided the original work is properly cited.

\section{INTRODUCTION}

Systematics in plant biodiversity is very important to know about the study of species diversity and its conservation, extinction and evolution, threats to biodiversity and biogeographic regions, exploration and other ecological services ${ }^{1}$. Diversity basically means total number of species within specific geographic areas, whether alien or native, which is the identity and source of vegetation and plant natural resources. Agriculture, deforestation, overgrazing, over-browsing, natural disasters and anthropogenic interaction are mainly affected by the plant 
resources $^{2}$. The family Poaceae (Grass family) is the $4^{\text {th }}$ spermatophyte family having 11000 species and 800 genera worldwide. However, in Pakistan, a total of 158 genera and 492 species are available ${ }^{3}$. Arundinoideae, Oryzoideae, Bambusoideae, Eragrostoideae and Panicoideae are important subfamilies of Gramineae 4 . Grasses can survive well to exposed, peripheral and commonly disturbed habitats, and it exist everywhere. Grass is characterized by the presence of its grain or caryopsis, and inflorescence having spikelet with a palea and lemma ${ }^{5}$. Overgrazing, over-browsing, water-logging, soil erosion, over-hunting and non-manageable agrarian methods have turned out to be significant dangers to biodiversity. The over-grazing of these fields has brought about harm to suitable grass and pea species. Due to continuous and heavy grazing, the pastures and forestareas have deteriorated. The interaction of natural grassland population and its structure with regards to its well-known ecological aspects have not been identified yet $^{6}$. Living organisms on the planet mostly depend on grains like maize, rice, oats, sugarcane, wheat and rye for their substantial need as energy crops. In addition, domesticated ruminants depend on these grasses. Moreover, those weeds growing on the agronomic land are also belong to the family Poaceae. This floristic survey provides a baseline to taxonomists to work on different aspects of plant wealth and status for their exploitation on systematic approaches and scientific basis.

A large number of livestock depends on natural vegetation through over-grazing and over-browsing, for fodder. Moreover, fuel, tubers and other edible parts are received from the forest in addition to the cultivation of some seasonal agricultural crops like maize and wheat in small areas ${ }^{7}$. Grasses are among the most adaptable life forms ${ }^{8}$. The flower of grasses suggests that they are tristaminate, bi-stigmatic and lodiculated in which stamens exist in two different cycles-two lateral from inner whorl and single outer frontal whorl 9 . The information with regards to species configuration of a region is considered to be an incentive for any phyto-geographical, environmental, ecological, and managing events. The floristic structure reveals the variety of natural resources which are affected by different abiotic and biotic stresses viz. over-grazing, over-browsing, deforestation, soil texture, soil corrosion and improper collection of these grasses in the area $^{10}$. Different authors have studied grasses time to time. In Pakistan most of the study on grasses was done by Chaudhari et al.,11 who studied ethnobotany of grasses from Thal desert, Pakistan, while Mehmood et al., ${ }^{7}$ documented floristic list of grasses from Tor Ghar, Pakistan in which 73 species with 54 genera and 6 subfamilies were included. Samreen et al., ${ }^{12}$ also conducted the similar study on grasses from district Bannu, Pakistan.

\section{Objectives of the Research}

The main focus of our study was the documentation and exploration of wild grasses from the area, which might help in future researches.

A floristic survey was conducted helpful in identifying plant wealth and status for their exploitation on systematic approaches and scientific basis.

\section{MATERIALS AND METHODS}

\section{Site Details and Duration of the Study}

The current survey was carried out in the District Charsadda in the blooming and flowering season during 2017-18 to assemble diverse grass species from the selected area. District Charsadda lies between $34^{\circ} 03^{\prime}$ to $34^{\circ} 28^{\prime}$ North latitude and $71^{\circ} 28^{\prime}$ to $71^{\circ} 33^{\prime}$ East longitude. Charsadda being the geographic center lies about $282 \mathrm{~m}$ above sea level, coveres an area of 996 square. $\mathrm{km}^{2}$. The annual precipitation rate is $460.0 \mathrm{~mm}$, whereas the hottest month is June $\left(44^{\circ} \mathrm{C}\right)$, the coldest month is January $\left(5^{\circ} \mathrm{C}-10^{\circ} \mathrm{C}\right)$ and the wettest month is February. Charsadda is surrounded by four districts and one tribal area, on the East is district Mardan, on the North is Malakand, on the South is Peshawar and Nowshera districts and Mohmand Agency on the West ${ }^{13}$.

\section{Dominant Flora of the Area}

District Charsadda has a unique type of flora which includes a lot of green land vegetation. This District has different types of vegetation comprising herbs, trees, lianas, shrubs and climbers. Among the trees, diverse floral plant species exist, which belong to different genera and families. In selected areas, Morus alba, Dilbergia sissoo, Acacia modesta, Morus nigra, Morus laevigata, Populus ciliate, Acacia nilotica, Melia azedarach, 
Eucalyptus lanceolata, Bombax ceiba, Ficus carica, Citrus medica, Ficus elastica, Prunus domestica, Ailanthus altissima and Ziziphus jujuba are used as fuel, food, medicine and for making furniture etc.

\section{Field Methodology:}

\section{Collection, Preservation and Identification of Sample Grasses}

Grass specimens were collected, dried in the shade for two weeks, preserved, mounted and then identified with the assistance of Pakistans' flora (Nasir \& Ali14; Stewart ${ }^{15}$; Ali \& Nasir ${ }^{16}$; Ali \& Qaiser ${ }^{17}$ ) and other literature (Raunkiaer ${ }^{18}$; Hussain ${ }^{19}$ ). Habitat class and life cycle of each specimen were examined. Abundant classes were determined through ACFOR scale to describe species abundance in a given area. Quantitative ecological techniques were applied to determine various ecological attributes. In biological spectrum, each ecological parameter was categorized as followed by Raunkiaer ${ }^{18}$; Hussain ${ }^{19}$.

\section{RESULTS AND DISCUSSION}

Poaceae is an important grass family with great economic importance as it contains crops like Oryza sativa, Triticum aestivum, Zea maize, Hordeum vulgare, Avena sativa and Pennesitum typhoides. It is one of the leading families of Angiospermic plants growing in every part of the earth's crust. The current work presents a total of 51 taxa with 34 genera, 5 subfamilies and 11 tribes were collected from the District Charsadda (Table 1, 3 \& 4). Among them, 3 species belonged to the genus Eragrostis (8.823\%), Saccharum (8.823\%) and Setaria (8.823\%) respectively. Genera Avena, Brachiaria, Bromus, Cymbopogon, Digitaria, Echinochloa, Hordeum, Phragmites, Poa, Polypogon and Sorghum had 2 species (5.882\%) each. While the remaining genera Alepecurous, Apluda, Arundo, Acrachne, Cenchrus, Cynodon, Dactyloctenium, Desmostachya, Dichantium, Eleusine, Imperata, Leptochloa, Oryza, Paspalum, Pennesetum, Phalaris, Rostraria, Stipa, Triticum and Zea had 1 species (2.941\%) each. The dominant genera were Eragrostis, Saccharum and Setaria 3 taxa followed by Avena, Brachiaria and Bromus 2 taxa followed by Alepecurous, Apluda and Arundo had only one taxa. The subfamily Arundinoideae had only 1 tribe and 3 taxa, Chloridoideae had 2 tribes
Cynodonteae and Eragrosteae, the tribe Cynodonteae had 1 taxa, while the tribe Eragrosteae had 7 taxa, the subfamily Oryzoideae had 1 tribe and 1 taxa, the subfamily Panicoideae had 2 tribes Andropogoneae and Paniceae, the tribe Andropogoneae had 11 taxa, while the tribe Paniceae had 12 taxa, the subfamily Pooideae had 5 tribes viz. Aveneae, Bromeae, Poeae, Stipeae and Triticeae. The tribe was first documented by Barthélemy Charles Joseph Dumortier from Belgian in 1823 who named it Triticeae ${ }^{20}$. The Tribe Poeae had 5 taxa, the tribe Aveneae had 4 taxa, the tribe Triticeae had 3 taxa, the tribe Bromeae had 2 taxa and the tribe Stipeae had only 1 taxa. The dominant subfamily was Pooideae having 5 tribes followed by Chloridoideae and Panicoideae having 2 tribes each, followed by Arundinoideae and Oryzoideae having only 1 tribe each. Various scientists also conducted the same study from different regions of Pakistan (Ahmad et al., ${ }^{21}$, Mehmood et al., ${ }^{7}$, Rafay et al., ${ }^{22}$, Ahmad et al., ${ }^{23}$ and Ullah et al., ${ }^{24}$ ). The biological spectrum is the climatic indicator of an area and can be considered as a symbolic representation of deep and shallow or soft and harsh climate of an area. Therefore, it plays an important role in vegetation description ${ }^{25}$.

Biological spectrum (Table 1 \& 2-A) showed that in the Life form class, Therophyte was the leading life form class with 32 species (62.74\%), followed by Hemicryptophyte having 13 species $(25.49 \%)$, further followed by Chaemophyte and Geophyte with 3 species (5.882\%) each (Figure 1).

In the Leaf size class, microphyll had the largest number of species i.e., 24 (47.05\%) followed by nanophyll having 14 species (27.45\%), mesophyll with 8 species $(15.68 \%)$, followed by macrophyll 3 species $(5.882 \%)$ and leptophyll 2 species (3.921\%), respectively (Table $1 \&$ 2-B, Figure 2). The Therophytic life form can survive with adverse environmental conditions and penetrating anthropogenic disturbance, whereas another affecting factor includes high grazing pressure. Climatic and anthropogenic disturbance in a specific area represents variation in both the Leaf size class and Life form class ${ }^{25}$. Therophytes and microphylls dominancy indicates the harshness, warmth and climatic environment of the area and the duration of the winter period. Only the cone-bearing and sclerophyllous plants sustain their life span is an 
evergreen area and remain vigorous during the winter season ${ }^{26}$. Our findings are in line with that of Ali et al., ${ }^{27}$; Khan and Shah ${ }^{28}$ and Sher et al., ${ }^{29}$, who also proved the dominancy of the therophytic and microphyllus class in his study. Badshah et al., ${ }^{2}$ stated that species composition is an ecological expression that not only reveals the proper description of semiarid regions, but also gives a lot of quantitative exploration of the vegetation resources that must be needed. Thus, species composition is a basic parameter of plant phenology and conservation of plants in any specific area. The ecological studies of plants biospectrum, habit, habitat, flowering season, phenology and its ethnoecological amplitude are to be worked out. The richness of the number of species of Asteraceae and Poaceae is due to their extensive conservation in a geographical area. The Habitat class (Table 1 \& 2-C, Figure 3) showed that 32 species $(62.74 \%)$ were terrestrial and 19 species (37.25\%) were amphibious in our study. The Life cycle class (Table 1 \& 2-D, Figure 4) depicted that 33 species (64.70\%) were annual, 17 species (33.33\%) were perennial and 1 specie (1.960\%) was annual or perennial. The Abundant class was determined by the ACFOR scale used to describe species abundance in a given area; 26 species (50.98\%) were occasional, 12 species (23.52\%) were frequent, 7 species $(13.72 \%)$ were rare, 5 species $(9.803 \%)$ were common and only 1 species Cynodon dactylon (1.960\%) was abundant in whole area and present everywhere in research area in every season (Table $1 \&$ 2-E, Figure 5). The Palatability class (Table 2-F \& 5, Figure 6) showed that 35 species $(68.62 \%)$ were highly palatable followed by less-palatable 11 species $(21.56 \%)$, non-palatable 2 species (3.921\%) and moderately palatable 3 species $(5.882 \%)$ (Table 5). Khan and Shah $^{28}$ also gave the statement of palatability from the district Mardan. Thus, the taxonomic study of Poaceae suggested that it is very homogenous taxon and field recognized family, the stem is usually rounded, hollow internodes are present, 2ranked leaves, open or sometimes closed sheath, ligule present, floret (flower) containing two bracts palea and lemma, 0-3 lodicules in perianth, the stamens usually 3 in number and the fruit is grain or caryopsis ${ }^{30}$. Zereen et al., ${ }^{31}$ studied the uses of grasses from central Punjab. A total of 51 species and 46 genera of grasses were reported from the area. Our findings also agree with that of Perveen and Qaiser ${ }^{30}$, Osman et al., ${ }^{32}$ Yen and Yang20, who also reported grass species from various parts of the country. As it is clear that no prior work has been done on the grasses in the Charsadda district, hence the current study is a benchmark for future researches.

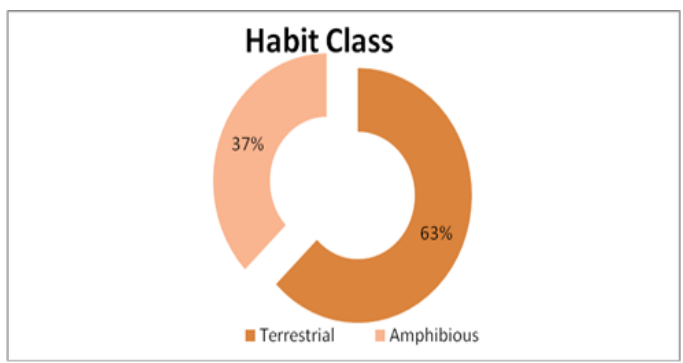

Figure 1. Percentage of Habitat Class of the Flora.

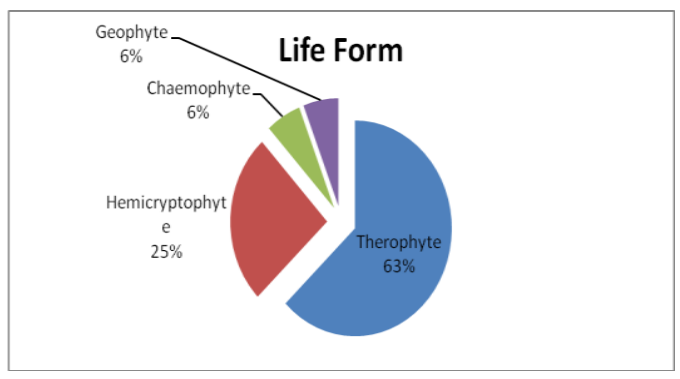

Figure 2. Percentage of Life Form Class.

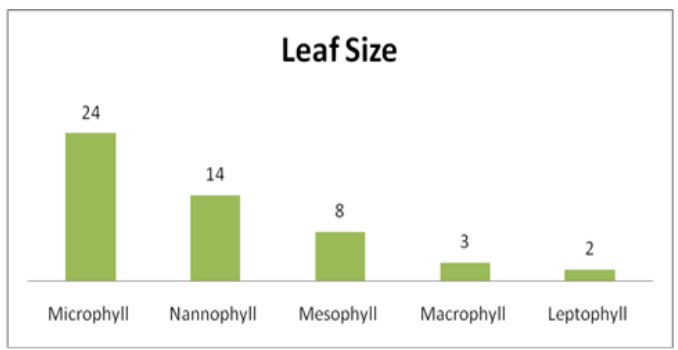

Figure 3. Graph Showing the Proportions of Leaf Size Spectrum.

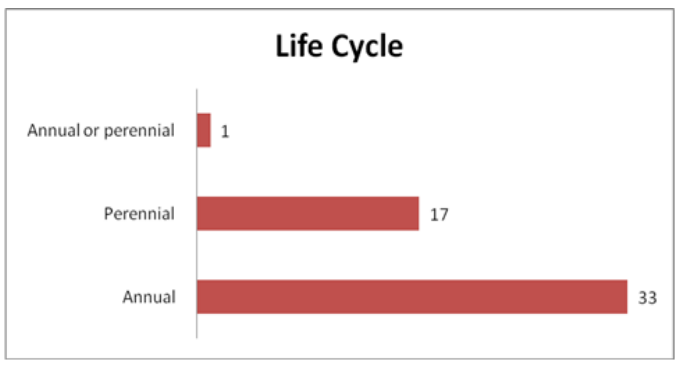

Figure 4. Bar-Graph Showing the Proportions of Life Span. 


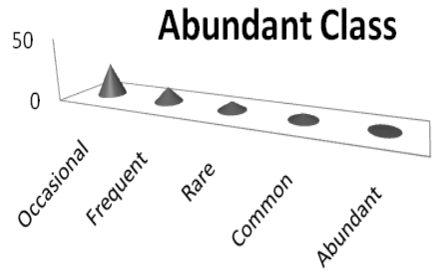

Figure 5. Showing the Proportions Abundant Class.

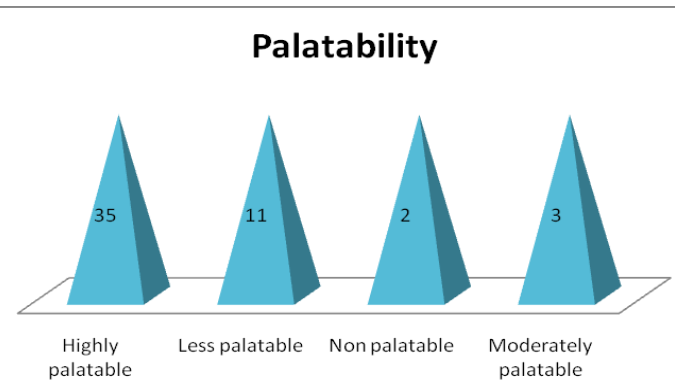

Figure 6. Graph Showing Palatability Status of Plants.

\section{Table 1. Biological Spectrum of Some Palatable Grasses of District Charsadda.}

\begin{tabular}{|c|c|c|c|c|c|c|c|}
\hline $\begin{array}{l}\text { S. } \\
\text { No. }\end{array}$ & Botanical Name & English Name & Habitat & Life Cycle & $\begin{array}{c}\text { Abundance } \\
\text { Class }\end{array}$ & $\begin{array}{l}\text { Life } \\
\text { Form }\end{array}$ & $\begin{array}{l}\text { Leaf } \\
\text { Size }\end{array}$ \\
\hline 1. & $\begin{array}{c}\text { Alopecurus myosuroides } \\
\text { Hudson. }\end{array}$ & Slender Foxtail & Amphibious & Annual & Frequent & Th & Mes \\
\hline 2. & Apluda mutica L. & Mauritian grass & Terrestrial & Perennial & Frequent & Th & Mic \\
\hline 3. & Arundo donax L. & Giant reed & Amphibious & Perennial & Frequent & Geo & Mes \\
\hline 4. & $\begin{array}{c}\text { Acrachne racemosa } \\
\text { Heyne ex Roem. Schult }\end{array}$ & Goose grass. & Terrestrial & Annual & Rare & $\mathrm{H}$ & Mic \\
\hline 5. & Avena fatua $\mathrm{L}$. & Oat & Amphibious & Annual & Frequent & Th & Mic \\
\hline 6. & Avena sativa $\mathrm{L}$. & Wild oat & Amphibious & Annual & Occasional & Th & $\mathrm{Na}$ \\
\hline 7. & $\begin{array}{c}\text { Brachiaria ramosa (Linn.) } \\
\text { Stapf }\end{array}$ & Browntop Millet & Terrestrial & Annual & Occasional & Th & Mic \\
\hline 8. & $\begin{array}{l}\text { Brachiaria reptans (Linn.) } \\
\text { Gardner \& Hubbard. }\end{array}$ & Running Grass & Amphibious & Annual & Frequent & Th & Mic \\
\hline 9. & Bromus catharticus Vahl. & Rescue grass & Terrestrial & Perennial & Occasional & Th & Mic \\
\hline 10. & Bromus pectinatus Thunb. & Brome grass & Terrestrial & Annual & Occasional & Th & Mes \\
\hline 11. & Cenchrus ciliaris L. & $\begin{array}{c}\text { Buffel grass, } \\
\text { kolukkatai }\end{array}$ & Terrestrial & Perennial & Occasional & Th & Mes \\
\hline 12. & $\begin{array}{l}\text { Cymbopogon citratus } \\
\text { (DC.) Stapf. }\end{array}$ & $\begin{array}{c}\text { Lemon grass or oil } \\
\text { grass }\end{array}$ & Terrestrial & Perennial & Rare & $\mathrm{H}$ & Mes \\
\hline 13. & $\begin{array}{c}\text { Cymbopogon jwarancusa } \\
\text { (Jones) Schult }\end{array}$ & Oil grass & Terrestrial & Perennial & Frequent & $\mathrm{H}$ & $\mathrm{Na}$ \\
\hline 14. & $\begin{array}{c}\text { Cynodon dactylon (L.) } \\
\text { Pers }\end{array}$ & Bermuda grass & Terrestrial & Perennial & Abundant & $\mathrm{H}$ & Lep \\
\hline 15. & $\begin{array}{l}\text { Dactyloctenium aegyptium } \\
\text { (L.) Willd }\end{array}$ & $\begin{array}{l}\text { crow foot } \\
\text { grass }\end{array}$ & Amphibious & Annual & Occasional & Th & Mic \\
\hline 16. & $\begin{array}{c}\text { Desmostachya } \\
\text { bipinnata (L.) Stapf. }\end{array}$ & Salt reed-grass & Amphibious & Perennial & Common & $\mathrm{H}$ & $\mathrm{Na}$ \\
\hline 17. & $\begin{array}{l}\text { Dichanthium annulatum } \\
\text { (Forssk.) Stapf }\end{array}$ & $\begin{array}{l}\text { Hindi grass, Sheda } \\
\text { grass }\end{array}$ & Amphibious & Perennial & Common & $\mathrm{H}$ & $\mathrm{Na}$ \\
\hline 18. & $\begin{array}{c}\text { Digitaria ciliaris (Retz.) } \\
\text { Koeler. }\end{array}$ & Crab grass & Amphibious & Annual & Occasional & $\mathrm{H}$ & Mic \\
\hline 19. & $\begin{array}{l}\text { Digitaria sanguinolenta } \\
\text { Edgew. ex Aitch. }\end{array}$ & Hairy crabgrass, & Amphibious & Annual & Rare & $\mathrm{H}$ & Mic \\
\hline 20. & $\begin{array}{c}\text { Echinochloa colona (L.) } \\
\text { Link }\end{array}$ & Jungle rice & Amphibious & Annual & Occasional & Th & $\mathrm{Na}$ \\
\hline 21. & $\begin{array}{c}\text { Echinochloa crus-gallis } \\
\text { (L.) Beauv }\end{array}$ & & Terrestrial & Annual & Occasional & Th & $\mathrm{Na}$ \\
\hline 22. & $\begin{array}{l}\text { Eleusine indica (L.) } \\
\text { Garetn. }\end{array}$ & Indian goose grass & Amphibious & Annual & Occasional & Th & Mes \\
\hline
\end{tabular}




\begin{tabular}{|c|c|c|c|c|c|c|c|}
\hline 23 & $\begin{array}{c}\text { Eragrostis nigra Nees. Ex. } \\
\mathrm{S}\end{array}$ & Love grass & Amphibious & $\begin{array}{l}\text { Annual or } \\
\text { perennial }\end{array}$ & Occasional & Th & Mic \\
\hline 24. & $\begin{array}{c}\text { Eragrostis cilianensis (All.) } \\
\text { Lut. }\end{array}$ & Love grass & Terrestrial & Annual & Rare & $\mathrm{H}$ & $\mathrm{Na}$ \\
\hline 25. & Eragrostis minor Host. & $\begin{array}{l}\text { Pungent meadow } \\
\text { grass }\end{array}$ & Amphibious & Annual & Occasional & Th & $\mathrm{Na}$ \\
\hline 26. & Hordeum murinum L. & False barley & Terrestrial & Annual & Occasional & Th & $\mathrm{Na}$ \\
\hline 27. & Hordeum vulgare L. & Barley, barley corn & Terrestrial & Annual & Frequent & Th & Mic \\
\hline 28. & $\begin{array}{c}\text { Imperata cylindrica (L.) P. } \\
\text { Beauv. }\end{array}$ & Cogon grass & Amphibious & Perennial & Occasional & Geo & Lep \\
\hline 29. & Leptochloa panicea Retz. & $\begin{array}{l}\text { Mucronate } \\
\text { Sprangletop }\end{array}$ & Terrestrial & Annual & Occasional & Th & Mic \\
\hline 30. & Oryza sativa L. & $\begin{array}{l}\text { Asian Rice, weedy } \\
\text { rice }\end{array}$ & Amphibious & Annual & Frequent & Th & Mic \\
\hline 31. & $\begin{array}{l}\text { Paspalum paspalodes } \\
\text { (Michx.) scribner. }\end{array}$ & $\begin{array}{l}\text { Water couch, } \\
\text { Knotgrass }\end{array}$ & Terrestrial & Perennial & Frequent & $\mathrm{H}$ & Mic \\
\hline 32. & $\begin{array}{l}\text { Pennesetum typhoides } \\
\text { (Burm.f.) Stapf. }\end{array}$ & Pearl millet & Terrestrial & Annual & Occasional & Th & Mic \\
\hline 33. & Phalaris minor Retz. & Canary grass & Terrestrial & Annual & Frequent & Th & $\mathrm{Na}$ \\
\hline 34. & $\begin{array}{l}\text { Phragmites australis } \\
\text { (Cav.) Trin. }\end{array}$ & Common reed & Amphibious & Perennial & Occasional & Geo & Mac \\
\hline 35. & $\begin{array}{l}\text { Phragmites karka (Retz.) } \\
\text { Trin. ex. Steud. }\end{array}$ & Nodding reed & Amphibious & Perennial & Occasional & $\mathrm{Ch}$ & Mes \\
\hline 36. & Poa annua L. & Annual blue grass & Terrestrial & Annual & Occasional & Th & $\mathrm{Na}$ \\
\hline 37. & Poa infirma H. B. K. & Early meadow-grass & Terrestrial & Annual & Occasional & Th & $\mathrm{Na}$ \\
\hline 38. & $\begin{array}{l}\text { Polypogon fugax } \\
\text { Ness.ex.Steud. }\end{array}$ & $\begin{array}{c}\text { Asia Minor Blue } \\
\text { grass }\end{array}$ & Terrestrial & Annual & Frequent & $\mathrm{H}$ & Mic \\
\hline 39. & $\begin{array}{c}\text { Polypogon monspeliensis } \\
\text { (L.) Desf }\end{array}$ & Rabbit foot grass & Terrestrial & Annual & Frequent & Th & Mic \\
\hline 40. & $\begin{array}{c}\text { Rostraria cristata (L.) } \\
\text { Tzvelev }\end{array}$ & $\begin{array}{l}\text { Mediterranean hair } \\
\text { grass }\end{array}$ & Terrestrial & Annual & Occasional & Th & Mic \\
\hline 41. & $\begin{array}{l}\text { Saccharum griffthii Munro } \\
\text { ex Boiss }\end{array}$ & Broom sedge & Terrestrial & Perennial & Rare & $\mathrm{H}$ & Mac \\
\hline 42. & Saccharum officinarum L. & Sugar cane & Terrestrial & Perennial & Common & $\mathrm{Ch}$ & Mic \\
\hline 43. & $\begin{array}{c}\text { Saccharum spontaneum } \\
\text { L. }\end{array}$ & Wild sugarcane & Terrestrial & Perennial & Occasional & $\mathrm{Ch}$ & Mac \\
\hline 44. & $\begin{array}{l}\text { Setaria pumila (Poir.) } \\
\text { Roem. \&Schult. }\end{array}$ & Yellow foxtail grass & Terrestrial & Annual & Occasional & Th & Mic \\
\hline 45. & $\begin{array}{c}\text { Setaria verticillata (L.) P. } \\
\text { Beauv }\end{array}$ & Fox tail & Terrestrial & Annual & Rare & Th & $\mathrm{Na}$ \\
\hline 46. & $\begin{array}{c}\text { Setaria viridis (L.) P. } \\
\text { Beauv }\end{array}$ & Green bristle grass & Amphibious & Annual & Occasional & Th & $\mathrm{Na}$ \\
\hline 47. & $\begin{array}{c}\text { Sorghum bicolor (L.) } \\
\text { Moench. }\end{array}$ & Grain sorghum & Terrestrial & Annual & Occasional & Th & Mic \\
\hline 48. & $\begin{array}{c}\text { Sorghum halepense (L.) } \\
\text { Pers. }\end{array}$ & Johnson grass & Terrestrial & Perennial & Occasional & $\mathrm{H}$ & Mic \\
\hline 49. & Stipa capensis Thunb. & Cape rice grass & Terrestrial & Annual & Rare & Th & Mic \\
\hline 50. & Triticum aestivum L. & Common wheat & Terrestrial & Annual & Common & Th & Mic \\
\hline 51. & Zea mays L. & Corn & Terrestrial & Annual & Common & Th & Mes \\
\hline
\end{tabular}

Keys:

A. Life form: Th-Therophyte, H-Hemicryptophyte, Ch-Chaemeophyte, G-Geophyte.

B. Leaf size: Lep-Leptophyll, Na-Nanophyll, Mic-Microphyll, Mac-Macrophyll, Mes-Mesophyll 
Table 2. Ecological Physiognomies of Palatable Grasses of District Charsadda, Pakistan.

\begin{tabular}{|c|c|c|c|}
\hline S. No. & Parameters & No. of Taxa & Percentages \\
\hline A. & Life form & & \\
\hline 1. & Therophyte & 32 & 62.74 \\
\hline 2. & Hemicryptophyte & 13 & 25.49 \\
\hline 3. & Chaemophyte & 3 & 5.882 \\
\hline \multirow[t]{2}{*}{4.} & Geophyte & 3 & 5.882 \\
\hline & Total & 51 & 99.99 \\
\hline B. & Leaf size & & \\
\hline 1. & Microphyll & 24 & 47.05 \\
\hline 2. & Nanophyll & 14 & 27.45 \\
\hline 3. & Mesophyll & 8 & 15.68 \\
\hline 4. & Macrophyll & 3 & 5.882 \\
\hline \multirow[t]{2}{*}{5.} & Leptophyll & 2 & 3.921 \\
\hline & Total & 51 & 99.99 \\
\hline C. & Habitat class/Adaptation & & \\
\hline 1. & Terrestrial & 32 & 62.74 \\
\hline \multirow[t]{2}{*}{2.} & Amphibious & 19 & 37.25 \\
\hline & Total & 51 & 99.99 \\
\hline D. & Life cycle & & \\
\hline 1. & Annual & 33 & 64.70 \\
\hline 2. & Perennial & 17 & 33.33 \\
\hline \multirow[t]{2}{*}{3.} & Annual or perennial & 1 & 1.960 \\
\hline & Total & 51 & 99.99 \\
\hline E. & Abundant class & & \\
\hline 1. & Occasional & 26 & 50.98 \\
\hline 2. & Frequent & 12 & 23.52 \\
\hline 3. & Rare & 7 & 13.72 \\
\hline 4. & Common & 5 & 9.803 \\
\hline \multirow[t]{2}{*}{5.} & Abundant & 1 & 1.960 \\
\hline & Total & 51 & 99.99 \\
\hline F. & Palatability & & \\
\hline 1. & Highly palatable & 35 & 68.62 \\
\hline 2. & Less palatable & 11 & 21.56 \\
\hline 3. & Non palatable & 2 & 3.921 \\
\hline \multirow[t]{2}{*}{4.} & Moderately palatable & 3 & 5.882 \\
\hline & Total & 51 & 99.99 \\
\hline
\end{tabular}


Table 3. List of Genera and its Percentage.

\begin{tabular}{|c|c|c|c|}
\hline S. No. & Genera & No. of Taxa & Percentages \\
\hline 1. & Eragrostis & 3 & $8.823 \%$ \\
\hline 2. & Saccharum & 3 & $8.823 \%$ \\
\hline 3. & Setaria & 3 & $8.823 \%$ \\
\hline 4. & Avena & 2 & $5.882 \%$ \\
\hline 5. & Brachiaria & 2 & $5.882 \%$ \\
\hline 6. & Bromus & 2 & $5.882 \%$ \\
\hline 7. & Cymbopogon & 2 & $5.882 \%$ \\
\hline 8. & Digitaria & 2 & $5.882 \%$ \\
\hline 9. & Echinochloa & 2 & $5.882 \%$ \\
\hline 10. & Hordeum & 2 & $5.882 \%$ \\
\hline 11. & Phragmites & 2 & $5.882 \%$ \\
\hline 12. & Poa & 2 & $5.882 \%$ \\
\hline 13. & Polypogon & 2 & $5.882 \%$ \\
\hline 14. & Sorghum & 2 & $5.882 \%$ \\
\hline 15. & Apluda & 1 & $2.941 \%$ \\
\hline 16. & Alopecurus & 1 & $2.941 \%$ \\
\hline 17. & Arundo & 1 & $2.941 \%$ \\
\hline 18. & Acrachne & 1 & $2.941 \%$ \\
\hline 19. & Cenchrus & 1 & $2.941 \%$ \\
\hline 20. & Cynodon & 1 & $2.941 \%$ \\
\hline 21. & Desmostachya & 1 & $2.941 \%$ \\
\hline 22. & Dactyloctenium & 1 & $2.941 \%$ \\
\hline 23. & Dichanthium & 1 & $2.941 \%$ \\
\hline 24. & Eleusine & 1 & $2.941 \%$ \\
\hline 25. & Imperata & 1 & $2.941 \%$ \\
\hline 26. & Leptochloa & 1 & $2.941 \%$ \\
\hline 27. & Oryza & 1 & $2.941 \%$ \\
\hline 28. & Paspalum & 1 & $2.941 \%$ \\
\hline 29. & Pennesetum & 1 & $2.941 \%$ \\
\hline 30. & Phalaris & 1 & $2.941 \%$ \\
\hline 31. & Rostraria & 1 & $2.941 \%$ \\
\hline 32. & Stipa & 1 & $2.941 \%$ \\
\hline 33. & Triticum & 1 & $2.941 \%$ \\
\hline 34. & Zea & 1 & $2.941 \%$ \\
\hline Total & 34 & 51 & $99.99 \%$ \\
\hline
\end{tabular}


Table 4. Distribution of Sub Families, Tribes and Taxa.

\begin{tabular}{|c|c|c|c|}
\hline S. No. & Sub Family & Tribe & Taxa \\
\hline \multirow{3}{*}{1.} & \multirow{3}{*}{ Arundinoideae } & \multirow{3}{*}{ Arundineae } & Arundo donaxL \\
\hline & & & Phragmites australis(Cav.) Trin. exSteud \\
\hline & & & Phragmites karka (Retz.) Trin. ex. Steud \\
\hline \multirow{9}{*}{2.} & \multirow{9}{*}{ Chloridoideae } & Cynodonteae & Cynodon dactylon (L.) Pers. \\
\hline & & \multirow{8}{*}{ Eragrosteae } & Acrachne racemosa (B.Heyne ex Roth) Ohwi. \\
\hline & & & Dactyloctenium aegyptium (L.) Willd \\
\hline & & & Desmostachya bipinnata (L.) Stapf. \\
\hline & & & Eleusine indica (L.) Garetn. \\
\hline & & & Eragrostis nigraNees. Ex. Steud \\
\hline & & & Eragrostis cilianensis (All.) Lut. \\
\hline & & & Eragrostis minor Host. \\
\hline & & & Leptochloa panicea Retz. \\
\hline 3. & Oryzoideae & Oryzeae & Oryza sativa L. \\
\hline \multirow{23}{*}{4.} & \multirow{23}{*}{ Panicoideae } & \multirow{11}{*}{ Andropogoneae } & Apluda mutica L. \\
\hline & & & Cymbopogon citratus (DC.) Stapf. \\
\hline & & & Cymbopogon jwarancusa (Jones) Schult. \\
\hline & & & Dichanthium annulatum (Forssk.) Stapf. \\
\hline & & & Imperata cylindrica (L.) P. Beauv \\
\hline & & & Saccharum griffthi Munro ex Boiss. \\
\hline & & & Saccharum officinarum L. \\
\hline & & & Saccharum spontaneum L. \\
\hline & & & Sorghum bicolor (L.) Moench \\
\hline & & & Sorghum halepense (L.) Pers \\
\hline & & & Zea mays L. \\
\hline & & \multirow{12}{*}{ Paniceae } & Brachiaria ramosa L. \\
\hline & & & Brachiaria reptans (L.) Gardner \& Hubbard \\
\hline & & & Cenchrus ciliaris L. \\
\hline & & & Digitaria ciliaris (Retz.) Koeler. \\
\hline & & & Digitaria sanguinolenta Edgew. ex Aitch. \\
\hline & & & Echinochloa colona (L.) Link \\
\hline & & & Echinochloa crus-gallis (L.) Beauv \\
\hline & & & Paspalum paspalodes (Michx.) scribner \\
\hline & & & Pennesetum typhoides (Burm.f.) Stapf \& C.E. Hubb. \\
\hline & & & Setaria pumila (Poir.) Roem. \& Schult. \\
\hline & & & Setaria verticillata (L.) P. Beauv \\
\hline & & & Setaria viridis (L.) P. Beauv \\
\hline \multirow{15}{*}{5.} & \multirow{15}{*}{ Pooideae } & \multirow{4}{*}{ Aveneae } & Avena fatua $\mathrm{L}$. \\
\hline & & & Avena sativa L. \\
\hline & & & Polypogon fugax Ness.ex.Steud \\
\hline & & & Polypogon monspeliensis (L.) Desf \\
\hline & & \multirow[t]{2}{*}{ Bromeae } & Bromus catharticus Vahl. \\
\hline & & & Bromus pectinatus Thunb. \\
\hline & & \multirow{5}{*}{ Poeae } & Alopecurus myosuroides Hudson. \\
\hline & & & Phalaris minor Retz. \\
\hline & & & Poa annua L. \\
\hline & & & Poa infirma H. B. K. \\
\hline & & & Rostraria cristata (L.) Tzvelev \\
\hline & & Stipeae & Stipa capensisThunb. \\
\hline & & \multirow{3}{*}{ Triticeae } & Hordeum murinum $\mathrm{L}$. \\
\hline & & & Hordeum vulgare L. \\
\hline & & & Triticum aestivum L. \\
\hline
\end{tabular}

Source: Gould \& Shaw ${ }^{33}$, (1983) 
Table 5. Botanical Taxa and its Common Names, Palatability and Distribution Pattern of Weedy Grasses in Pakistan and in the World.

\begin{tabular}{|c|c|c|c|c|c|}
\hline S. No. & Taxon & Common Name & Palatability & $\begin{array}{c}\text { Distribution Pattern in } \\
\text { Pakistan }\end{array}$ & Distribution Pattern in World \\
\hline 1. & $\begin{array}{c}\text { Alopecurus } \\
\text { myosuroides Hudson }\end{array}$ & Slender Foxtail & $\begin{array}{l}\text { Highly } \\
\text { palatable }\end{array}$ & $\begin{array}{c}\text { Baluchistan, Khyber } \\
\text { PakhtunKhwa \& Kashmir }\end{array}$ & $\begin{array}{c}\text { Europe, Asia, North America and } \\
\text { other temperate regions of the } \\
\text { world }\end{array}$ \\
\hline 2. & Apluda mutica L. & Mauritian grass & $\begin{array}{l}\text { Highly } \\
\text { palatable }\end{array}$ & $\begin{array}{c}\text { Sind, Punjab, Khyber } \\
\text { PakhtunKhwa \& Kashmir }\end{array}$ & $\begin{array}{c}\text { Tropical Asia, new Caledonia, } \\
\text { Oman, new Guinea and } \\
\text { throughout Australia }\end{array}$ \\
\hline 3. & Arundo donax L. & Giant reed & $\begin{array}{l}\text { Less } \\
\text { palatable }\end{array}$ & $\begin{array}{c}\text { Baluchistan, Punjab, Khyber } \\
\text { PakhtunKhwa \& Kashmir }\end{array}$ & $\begin{array}{c}\text { Eastwards to Burma; } \\
\text { Mediterranean regions and } \\
\text { North Africa and introduced into } \\
\text { new World }\end{array}$ \\
\hline 4. & $\begin{array}{c}\text { Acrachne racemosa } \\
\text { Heyne ex Roem. \& } \\
\text { Schult } \\
\end{array}$ & Goose grass & $\begin{array}{l}\text { Highly } \\
\text { palatable }\end{array}$ & $\begin{array}{c}\text { Punjab, Khyber PakhtunKhwa } \\
\text { \& Kashmir }\end{array}$ & $\begin{array}{l}\text { Tropical part of Australia, } \\
\text { southeast Asia and Africa }\end{array}$ \\
\hline 5. & Avena fatua $\mathrm{L}$. & Oat & $\begin{array}{l}\text { Highly } \\
\text { palatable }\end{array}$ & $\begin{array}{l}\text { Khyber PakhtunKhwa and } \\
\text { Northern Punjab }\end{array}$ & $\begin{array}{c}\text { Central Asia, Europe, USA, } \\
\text { Mississippi, Tennesseand } \\
\text { Australia }\end{array}$ \\
\hline 6. & Avena sativa L. & Wild oat & $\begin{array}{c}\text { Highly } \\
\text { palatable }\end{array}$ & $\begin{array}{l}\text { Khyber PakhtunKhwa and } \\
\text { Punjab }\end{array}$ & $\begin{array}{c}\text { Throughout Europe, Asia and } \\
\text { northwest Africa }\end{array}$ \\
\hline 7. & $\begin{array}{c}\text { Brachiaria } \\
\text { ramosa (Linn.) Stapf }\end{array}$ & Browntop Millet & $\begin{array}{l}\text { Highly } \\
\text { palatable }\end{array}$ & $\begin{array}{l}\text { Kashmir, Punjab, Baluchistan, } \\
\text { Sind, Khyber PakhtunKhwa }\end{array}$ & $\begin{array}{c}\text { Tropical Asia, South Africa, } \\
\text { Senegal, Yemen, Rhodesia and } \\
\text { Malawi }\end{array}$ \\
\hline 8. & $\begin{array}{c}\text { Brachiaria reptans } \\
\text { (Linn.) Gardner \& } \\
\text { Hubbard. }\end{array}$ & Running Grass & $\begin{array}{l}\text { Highly } \\
\text { palatable }\end{array}$ & $\begin{array}{l}\text { Khyber PakhtunKhwa, Sind, } \\
\text { Baluchistan \& Punjab }\end{array}$ & $\begin{array}{c}\text { Tropical Asia and throughout the } \\
\text { tropics }\end{array}$ \\
\hline 9. & $\begin{array}{c}\text { Bromus } \\
\text { catharticus Vahl. }\end{array}$ & Rescue grass & $\begin{array}{l}\text { Highly } \\
\text { palatable }\end{array}$ & $\begin{array}{l}\text { Punjab \& Khyber } \\
\text { PakhtunKhwa }\end{array}$ & $\begin{array}{c}\text { Native to South America, } \\
\text { Europe, Australia and North } \\
\text { America. }\end{array}$ \\
\hline 10. & $\begin{array}{l}\text { Bromus pectinatus } \\
\text { Thunb. }\end{array}$ & Brome grass & $\begin{array}{l}\text { Highly } \\
\text { palatable }\end{array}$ & $\begin{array}{c}\text { Khyber PakhtunKhwa, } \\
\text { Baluchistan, Punjab, Gilgit \& } \\
\text { Kashmir }\end{array}$ & $\begin{array}{l}\text { Through Ethiopia, Egypt and } \\
\text { Sudan, Arabia and Sinai, South } \\
\text { Africa, Afghanistan, Iran, } \\
\text { eastwards through India to } \\
\text { Europe and China. }\end{array}$ \\
\hline 11. & Cenchrus ciliaris L. & $\begin{array}{l}\text { Buffel grass, } \\
\text { kolukkatai }\end{array}$ & $\begin{array}{l}\text { Less } \\
\text { palatable }\end{array}$ & $\begin{array}{l}\text { Khyber PakhtunKhwa, } \\
\text { Baluchistan, Sind \& Punjab }\end{array}$ & $\begin{array}{c}\text { Distributed throughout Africa, } \\
\text { extending through Middle East to } \\
\text { Arabia and to India }\end{array}$ \\
\hline 12. & $\begin{array}{l}\text { Cymbopogon citratus } \\
\text { (DC.) Stapf. }\end{array}$ & $\begin{array}{l}\text { Lemon grass or } \\
\text { oil grass }\end{array}$ & $\begin{array}{l}\text { Non- } \\
\text { palatable }\end{array}$ & $\begin{array}{l}\text { Khyber PakhtunKhwa and } \\
\text { Punjab }\end{array}$ & $\begin{array}{c}\text { North and Central America, Sri } \\
\text { Lanka, China, India, Pakistan, } \\
\text { Indonesia, Nigeria, Thailand, } \\
\text { Cameroon, Italy, Congo, Egypt, } \\
\text { Argentina, Brazil, Venezuela and } \\
\text { Papua Guinea. }\end{array}$ \\
\hline 13. & $\begin{array}{c}\text { Cymbopogon } \\
\text { jwarancusa (Jones) } \\
\text { Schult. } \\
\end{array}$ & Oil grass & $\begin{array}{l}\text { Less } \\
\text { palatable }\end{array}$ & $\begin{array}{l}\text { Khyber PakhtunKhwa, Sind, } \\
\text { Baluchistan, Punjab \& Gilgit }\end{array}$ & $\begin{array}{l}\text { Westwards to Iraq and Socotra } \\
\text { also in Nepal and Northwest } \\
\text { India }\end{array}$ \\
\hline 14. & $\begin{array}{l}\text { Cynodon dactylon (L.) } \\
\text { Pers }\end{array}$ & Bermuda grass & $\begin{array}{l}\text { Highly } \\
\text { palatable }\end{array}$ & $\begin{array}{c}\text { Sind, Khyber PakhtunKhwa, } \\
\text { Baluchistan, Punjab \& } \\
\text { Kashmir }\end{array}$ & $\begin{array}{l}\text { Warm temperate and Tropical } \\
\text { regions; Cosmopolitan }\end{array}$ \\
\hline 15. & $\begin{array}{c}\text { Dactyloctenium } \\
\text { aegyptium(L.) Willd }\end{array}$ & $\begin{array}{l}\text { Crow foot } \\
\text { Grass }\end{array}$ & $\begin{array}{l}\text { Highly } \\
\text { palatable }\end{array}$ & $\begin{array}{l}\text { KhyberPakhtunKhwa, Sind, } \\
\text { Punjab \& Kashmir }\end{array}$ & $\begin{array}{c}\text { Commonly spread in warm } \\
\text { temperate and tropical regions of } \\
\text { the world. }\end{array}$ \\
\hline 16. & $\begin{array}{c}\text { Desmostachya } \\
\text { bipinnata (L.) Stapf }\end{array}$ & Salt reed-grass & $\begin{array}{l}\text { Less } \\
\text { palatable }\end{array}$ & $\begin{array}{l}\text { Sind, Baluchistan, Punjab } \\
\text { Kashmir \& Khyber } \\
\text { PakhtunKhwa }\end{array}$ & $\begin{array}{c}\text { Through-out the Middle east to } \\
\text { Indo-China, tropical Africa and } \\
\text { North America }\end{array}$ \\
\hline
\end{tabular}




\begin{tabular}{|c|c|c|c|c|c|}
\hline 17. & $\begin{array}{c}\text { Dichanthium } \\
\text { annulatum (Forssk.) } \\
\text { Stapf. }\end{array}$ & $\begin{array}{l}\text { Hindi grass, } \\
\text { Sheda grass }\end{array}$ & $\begin{array}{l}\text { Highly } \\
\text { palatable }\end{array}$ & $\begin{array}{l}\text { Sind, Baluchistan, Punjab } \\
\text { Kashmir \& Khyber } \\
\text { PakhtunKhwa }\end{array}$ & $\begin{array}{l}\text { Tropical America, Middle East, } \\
\text { Kenya, Tanzania, southern } \\
\text { Africa and Senegal, Indonesia } \\
\text { and introduced to Australia. }\end{array}$ \\
\hline 18. & $\begin{array}{l}\text { Digitaria ciliaris(Retz.) } \\
\text { Koeler. }\end{array}$ & Summer grass & $\begin{array}{c}\text { Highly } \\
\text { palatable }\end{array}$ & $\begin{array}{l}\text { Sind, Punjab, Kashmir \& } \\
\text { Khyber PakhtunKhwa. }\end{array}$ & Throughout the Tropics. \\
\hline 19. & $\begin{array}{l}\text { Digitaria sanguinolenta } \\
\text { Edgew. Ex Aitch }\end{array}$ & $\begin{array}{c}\text { Hairy crabgrass, } \\
\text { Hairy finger } \\
\text { grass. } \\
\end{array}$ & $\begin{array}{l}\text { Highly } \\
\text { palatable }\end{array}$ & $\begin{array}{c}\text { Baluchistan, Khyber } \\
\text { PakhtunKhwa, Punjab, Gilgit } \\
\text { \& Kashmir }\end{array}$ & $\begin{array}{l}\text { Tropical and temperate, } \\
\text { specifically into the tropics. }\end{array}$ \\
\hline 20. & $\begin{array}{l}\text { Echinochloa colona } \\
\text { (L.) Link. }\end{array}$ & Jungle rice & $\begin{array}{l}\text { Highly } \\
\text { palatable }\end{array}$ & $\begin{array}{c}\text { Sind, Baluchistan, Punjab, } \\
\text { Khyber PakhtunKhwa \& } \\
\text { Kashmir }\end{array}$ & $\begin{array}{l}\text { Distributed throughout tropics } \\
\text { and sub tropics }\end{array}$ \\
\hline 21. & $\begin{array}{l}\text { Echinochloa crus-galli } \\
\text { (L.) Beauv. }\end{array}$ & $\begin{array}{l}\text { Cockspur grass, } \\
\text { water grass }\end{array}$ & $\begin{array}{c}\text { Highly } \\
\text { palatable }\end{array}$ & $\begin{array}{c}\text { Sind, Baluchistan, Punjab, } \\
\text { Khyber PakhtunKhwa, Gilgit \& } \\
\text { Kashmir }\end{array}$ & $\begin{array}{l}\text { Confined to the Subtropical and } \\
\text { temperate regions of the world }\end{array}$ \\
\hline 22. & $\begin{array}{c}\text { Eleusine indica (L.) } \\
\text { Garetn. }\end{array}$ & $\begin{array}{l}\text { Indian goose } \\
\text { grass }\end{array}$ & $\begin{array}{c}\text { Highly } \\
\text { palatable }\end{array}$ & $\begin{array}{l}\text { Sind, Khyber PakhtunKhwa \& } \\
\text { Kashmir }\end{array}$ & $\begin{array}{l}\text { Present in Subtropical and } \\
\text { Tropical zones of the world }\end{array}$ \\
\hline 23. & $\begin{array}{c}\text { Eragrostis nigra Nees. } \\
\text { Ex. Steud }\end{array}$ & Love grass & $\begin{array}{c}\text { Highly } \\
\text { palatable }\end{array}$ & $\begin{array}{c}\text { Sind, Baluchistan, Punjab, } \\
\text { Khyber PakhtunKhwa }\end{array}$ & $\begin{array}{l}\text { Present in Subtropical and } \\
\text { Tropical zones of the world }\end{array}$ \\
\hline 24. & $\begin{array}{l}\text { Eragrostis cilianensis } \\
\text { (All.) Lut. Ex.F.T. }\end{array}$ & Love grass & $\begin{array}{l}\text { Highly } \\
\text { palatable }\end{array}$ & $\begin{array}{l}\text { Sind, Baluchistan, Punjab, } \\
\text { Khyber PakhtunKhwa \& } \\
\text { Kashmir }\end{array}$ & $\begin{array}{l}\text { Present in moist and Tropical } \\
\text { zones of the world }\end{array}$ \\
\hline 25. & Eragrostis minor Host. & $\begin{array}{l}\text { Pungent meadow } \\
\text { grass }\end{array}$ & $\begin{array}{l}\text { Highly } \\
\text { palatable }\end{array}$ & $\begin{array}{c}\text { Sind, Punjab, Gilgit, } \\
\text { Baluchistan, Khyber } \\
\text { PakhtunKhwa \& Kashmir } \\
\end{array}$ & $\begin{array}{l}\text { Subtropical and Warm temperate } \\
\text { regions of the world }\end{array}$ \\
\hline 26. & Hordeum murinum L. & False barley & $\begin{array}{c}\text { Less } \\
\text { palatable }\end{array}$ & $\begin{array}{c}\text { Khyber PakhtunKhwa \& } \\
\text { Kashmir }\end{array}$ & $\begin{array}{l}\text { Central Asia and Mediterranean } \\
\text { regions to China. }\end{array}$ \\
\hline 27. & Hordeum vulgare L. & $\begin{array}{l}\text { Barley, barley } \\
\text { corn }\end{array}$ & $\begin{array}{c}\text { Less } \\
\text { palatable }\end{array}$ & $\begin{array}{c}\text { Sind, Punjab, Khyber } \\
\text { PakhtunKhwa \& Kashmir }\end{array}$ & $\begin{array}{l}\text { Throughout most temperate } \\
\text { zones of the world }\end{array}$ \\
\hline 28. & $\begin{array}{l}\text { Imperata cylindrica (L.) } \\
\text { P. Beauv. }\end{array}$ & Cogon grass & $\begin{array}{l}\text { Moderately } \\
\text { palatable }\end{array}$ & $\begin{array}{c}\text { Sind, Baluchistan, Punjab, } \\
\text { Khyber PakhtunKhwa, Gilgit \& } \\
\text { Kashmir }\end{array}$ & $\begin{array}{l}\text { Mediterranean, Old World } \\
\text { tropics, Middle East and also in } \\
\text { Chile. }\end{array}$ \\
\hline 29. & $\begin{array}{c}\text { Leptochloa panicea } \\
\text { Retz. }\end{array}$ & $\begin{array}{l}\text { Mucronate } \\
\text { Sprangletop }\end{array}$ & $\begin{array}{l}\text { Moderately } \\
\text { palatable }\end{array}$ & $\begin{array}{c}\text { Sind, Punjab \& Khyber } \\
\text { PakhtunKhwa }\end{array}$ & $\begin{array}{l}\text { Natal; Sudan to Transvaal; } \\
\text { Tropical Asia and West Africa }\end{array}$ \\
\hline 30. & Oryza sativa L. & $\begin{array}{l}\text { Asian Rice, } \\
\text { weedy rice }\end{array}$ & $\begin{array}{l}\text { Less } \\
\text { palatable }\end{array}$ & $\begin{array}{c}\text { Sind, Punjab, Khyber } \\
\text { PakhtunKhwa and the chief } \\
\text { crop is in Kashmir (Liddar } \\
\text { Valley). }\end{array}$ & $\begin{array}{c}\text { Cultivated mostly in Central and } \\
\text { South America, southern } \\
\text { Europe, Africa, Asia and } \\
\text { Australia }\end{array}$ \\
\hline 31. & $\begin{array}{l}\text { Paspalum paspalodes } \\
\text { (Michx.) scribner }\end{array}$ & $\begin{array}{c}\text { Water } \\
\text { couch,Knotgrass }\end{array}$ & $\begin{array}{l}\text { Moderately } \\
\text { palatable }\end{array}$ & $\begin{array}{l}\text { Sind, Punjab, Khyber } \\
\text { PakhtunKhwa \& Kashmir }\end{array}$ & $\begin{array}{l}\text { Tropics and sub-tropics } \\
\text { throughout the world. }\end{array}$ \\
\hline 32. & $\begin{array}{l}\text { Pennesetum typhoides } \\
\text { (Burm.f.) Stapf \& C.E. } \\
\text { Hubb. }\end{array}$ & Pearl millet & $\begin{array}{l}\text { Highly } \\
\text { palatable }\end{array}$ & $\begin{array}{c}\text { Punjab, Sindh, Khyber } \\
\text { PakhtunKhwa and Balochistan }\end{array}$ & $\begin{array}{l}\text { South Africa from West to East; } \\
\text { It was Alien to India and later to } \\
\text { Australia, America and Brazil. }\end{array}$ \\
\hline 33. & Phalaris minor Retz. & Canary grass & $\begin{array}{c}\text { Highly } \\
\text { palatable }\end{array}$ & $\begin{array}{l}\text { Baluchistan, Punjab, Khyber } \\
\text { PakhtunKhwa \& Kashmir }\end{array}$ & Cosmopolitan \\
\hline 34. & $\begin{array}{l}\text { Phragmites australis } \\
\text { (Cav.) Trin. exSteud. }\end{array}$ & Common reed & $\begin{array}{c}\text { Non } \\
\text { palatable }\end{array}$ & $\begin{array}{l}\text { Khyber PakhtunKhwa, Punjab } \\
\text { \& Kashmir. }\end{array}$ & $\begin{array}{l}\text { Distributed throughout moderate } \\
\text { climate zones of New World and } \\
\text { Old World }\end{array}$ \\
\hline 35. & $\begin{array}{c}\text { Phragmites karka } \\
\text { (Retz.) Trin. ex. Steud. }\end{array}$ & Nodding reed & $\begin{array}{l}\text { Less } \\
\text { palatable }\end{array}$ & $\begin{array}{l}\text { Sind, Baluchistan, Punjab, } \\
\text { Khyber PakhtunKhwa, Gilgit \& } \\
\text { Kashmir }\end{array}$ & $\begin{array}{c}\text { Throughout Polynesia, Tropical } \\
\text { Africa, tropical Asia and northern } \\
\text { Australia. }\end{array}$ \\
\hline 36. & Poa annua L. & $\begin{array}{l}\text { Annual blue } \\
\text { grass }\end{array}$ & $\begin{array}{c}\text { Highly } \\
\text { palatable }\end{array}$ & $\begin{array}{c}\text { Baluchistan, Punjab, Khyber } \\
\text { PakhtunKhwa \& Kashmir }\end{array}$ & Cosmopolitan \\
\hline 37. & Poa infirma H. B. K. & $\begin{array}{l}\text { Early meadow- } \\
\text { grass }\end{array}$ & $\begin{array}{c}\text { Highly } \\
\text { palatable }\end{array}$ & $\begin{array}{c}\text { Punjab \& Khyber } \\
\text { PakhtunKhwa }\end{array}$ & $\begin{array}{l}\text { Central Asia, Himalayas of South } \\
\text { America; South Europe }\end{array}$ \\
\hline 38. & $\begin{array}{l}\text { Polypogon fugax Ness. } \\
\text { ex. Steud. }\end{array}$ & $\begin{array}{l}\text { Asia Minor Blue } \\
\text { grass }\end{array}$ & $\begin{array}{c}\text { Highly } \\
\text { palatable }\end{array}$ & $\begin{array}{l}\text { Baluchistan, Punjab, Khyber } \\
\text { PakhtunKhwa \& Kashmir }\end{array}$ & $\begin{array}{c}\text { Mainly in Himalayas of Burma } \\
\text { and Iraq }\end{array}$ \\
\hline
\end{tabular}




\begin{tabular}{|c|c|c|c|c|c|}
\hline 39. & $\begin{array}{c}\text { Polypogon } \\
\text { monspeliensis (L.) } \\
\text { Desf. }\end{array}$ & Rabbit foot grass & $\begin{array}{c}\text { Highly } \\
\text { palatable }\end{array}$ & $\begin{array}{l}\text { Sind, Baluchistan, Punjab, } \\
\text { Khyber PakhtunKhwa, Gilgit \& } \\
\text { Kashmir }\end{array}$ & $\begin{array}{l}\text { Introduced and adopted in most } \\
\text { warm temperate regions; } \\
\text { Mediterranean northwards zones } \\
\text { to British Isles; India and China; } \\
\text { North-east parts of south Africa }\end{array}$ \\
\hline 40. & $\begin{array}{l}\text { Rostraria cristata (L.) } \\
\text { Tzvelev. }\end{array}$ & $\begin{array}{l}\text { Mediterranean } \\
\text { hair grass }\end{array}$ & $\begin{array}{l}\text { Highly } \\
\text { palatable }\end{array}$ & $\begin{array}{l}\text { Baluchistan, Punjab, Khyber } \\
\text { PakhtunKhwa \& Kashmir }\end{array}$ & $\begin{array}{c}\text { Mediterranean region and } \\
\text { Northwest India; introduced in } \\
\text { North America and South Africa }\end{array}$ \\
\hline 41. & $\begin{array}{l}\text { Saccharum griffthii } \\
\text { Munro ex Boiss }\end{array}$ & Broom sedge & $\begin{array}{l}\text { Less } \\
\text { palatable }\end{array}$ & $\begin{array}{l}\text { Sind, Baluchistan, Punjab \& } \\
\text { Khyber PakhtunKhwa }\end{array}$ & $\begin{array}{l}\text { Asia-temperate; Asia-tropical; } \\
\text { western Asia and Arabia. } \\
\text { Afghanistan; India, Bangladesh; } \\
\text { Iran; Pakistan; Oman; Yemen; } \\
\text { Saudi Arabia and West Himalaya }\end{array}$ \\
\hline 42. & $\begin{array}{l}\text { Saccharum } \\
\text { officinarum. L. }\end{array}$ & Sugar cane & $\begin{array}{l}\text { Highly } \\
\text { palatable }\end{array}$ & $\begin{array}{c}\text { Sindh, Punjab and Khyber } \\
\text { PakhtunKhwa }\end{array}$ & $\begin{array}{l}\text { Throughout the tropical regions } \\
\text { while extending to temperate } \\
\text { regions of the world. }\end{array}$ \\
\hline 43. & $\begin{array}{c}\text { Saccharum } \\
\text { spontaneum L. }\end{array}$ & Wild sugarcane & $\begin{array}{l}\text { Less } \\
\text { palatable }\end{array}$ & $\begin{array}{c}\text { Sind, Punjab, Khyber } \\
\text { PakhtunKhwa, Gilgit \& } \\
\text { Kashmir }\end{array}$ & $\begin{array}{l}\text { Extensively scattered in the } \\
\text { tropical region of the Old World. }\end{array}$ \\
\hline 44. & $\begin{array}{l}\text { Setaria pumila (Poir.) } \\
\text { Roem. \& Schult. }\end{array}$ & $\begin{array}{l}\text { Yellow foxtail } \\
\text { grass }\end{array}$ & $\begin{array}{l}\text { Highly } \\
\text { palatable }\end{array}$ & $\begin{array}{l}\text { Sind, Baluchistan, Punjab, } \\
\text { Khyber PakhtunKhwa, Gilgit \& } \\
\text { Kashmir }\end{array}$ & $\begin{array}{l}\text { Introduced into warm temperate } \\
\text { and Tropical zones of the Old } \\
\text { World and North America. }\end{array}$ \\
\hline 45. & $\begin{array}{l}\text { Setaria verticillata (L.) } \\
\text { P. Beauv. }\end{array}$ & Fox tail & $\begin{array}{l}\text { Highly } \\
\text { palatable }\end{array}$ & $\begin{array}{c}\text { Sind, Baluchistan, Punjab, } \\
\text { Khyber PakhtunKhwa \& } \\
\text { Kashmir }\end{array}$ & $\begin{array}{l}\text { Confined to the warm temperate, } \\
\text { Tropical regions of the world. }\end{array}$ \\
\hline 46. & $\begin{array}{l}\text { Setaria viridis (L.) P. } \\
\text { Beauv. }\end{array}$ & $\begin{array}{l}\text { Green bristle } \\
\text { grass }\end{array}$ & $\begin{array}{l}\text { Highly } \\
\text { palatable }\end{array}$ & $\begin{array}{l}\text { Baluchistan, Punjab, Gilgit, } \\
\text { Kashmir and Khyber } \\
\text { PakhtunKhwa. }\end{array}$ & $\begin{array}{l}\text { Introduced to New World while } \\
\text { present also in cooler zones of } \\
\text { the Old World }\end{array}$ \\
\hline 47. & $\begin{array}{l}\text { Sorghum bicolor (L.) } \\
\text { Moench. }\end{array}$ & Grain sorghum & $\begin{array}{l}\text { Highly } \\
\text { palatable }\end{array}$ & $\begin{array}{l}\text { Sind, Baluchistan, Punjab, } \\
\text { Khyber PakhtunKhwa }\end{array}$ & $\begin{array}{l}\text { Radiating center/hotspot in } \\
\text { Africa, and is now widely } \\
\text { cultivated in subtropical and } \\
\text { tropical regions of the world. }\end{array}$ \\
\hline 48. & $\begin{array}{l}\text { Sorghum halepense } \\
\text { (L.) Pers. }\end{array}$ & Johnson grass & $\begin{array}{l}\text { Highly } \\
\text { palatable }\end{array}$ & $\begin{array}{l}\text { Sind, Punjab, Baluchistan, } \\
\text { Gilgit, Kashmir and Khyber } \\
\text { PakhtunKhwa. }\end{array}$ & $\begin{array}{l}\text { Mediterranean zones of } \\
\text { southwards to Madras and } \\
\text { Kashmir }\end{array}$ \\
\hline 49. & Stipa capensis Thunb. & Cape rice grass & $\begin{array}{l}\text { Highly } \\
\text { palatable }\end{array}$ & $\begin{array}{l}\text { Baluchistan, Punjab, Kashmir } \\
\text { and Khyber PakhtunKhwa }\end{array}$ & $\begin{array}{l}\text { Mediterranean region eastwards } \\
\text { to Northwest India; South Africa. }\end{array}$ \\
\hline 50. & Triticum aestivum L. & Common wheat & $\begin{array}{c}\text { Less } \\
\text { palatable }\end{array}$ & Throughout Pakistan & $\begin{array}{l}\text { Widely cultivated all over the } \\
\text { world. }\end{array}$ \\
\hline 51. & Zea mays L. & Corn/ Makki & $\begin{array}{l}\text { Highly } \\
\text { palatable }\end{array}$ & $\begin{array}{c}\text { Sind, Punjab, Khyber } \\
\text { PakhtunKhwa, Kashmir, Gilgit } \\
\text { and Balochistan }\end{array}$ & $\begin{array}{l}\text { Introduced to the Old World from } \\
\text { tropical America. }\end{array}$ \\
\hline
\end{tabular}

\section{CONCLUSION}

In the present study, we have reported 51 grass species belonging to 34 genera from 5 sub- families and 11 tribes for the first time from this area. The subfamily Panicoideae were more diverse sharing highest number of taxa, genera and tribes followed by subfamily Pooideae. Biological data represents that Therophyte was the dominant life form class, while the leaf size of Microphyll was dominant. Majority of our studied grasses were terrestrial with annual life span. The palatable grasses were documented due to its grazing in the area while fewer numbers of grasses were observed occasional in the area. Cynodon dactylon were recorded abundant in the study area. Therefore, it was concluded that due to uncontrolled grazing, over-exploitation and over-consumption are the major biotic threats which affects the diversity of the grasses in the area and also affects the livestock's production in the area. These present botanical endeavors provide a base line in the field of Agrostology for further study. 


\section{LIST OF ABBREVIATIONS}

ACFOR

Abundant Common Frequent

Occasional Rare

\section{REFERENCES}

1. Scotland RW, Wortley AH. How many species of seed plants are there? Taxon. 2003; 52(1):101-4.

2. Badshah L, Hussain F, Sher Z. Floristic inventory, ecological characteristics and biological spectrum of rangeland of District Tank, Pakistan. Pak J Bot. 2013; 45(4):1159-68.

3. Nasir E, Ali Sl, Cope TA. Poaceae: Flora of Pakistan (Eds.): 1982; 143:40-678.

4. Mabberley DI. The Plant Book Cambridge. Univ Press, Cambridge, New York. 2008.

5. Peterson PM. Poaceae (Gramineae). ELS John Wiley \& Sons, Ltd: Chichester. 2013.

6. Overbeck GE, Müller SC, Fidelis A, Pfadenhauer J, Pillar VD, Blanco CC, et al. Brazil's neglected biome: The South Brazilian Campos. Perspect Plant Ecol Syst. 2007; 9(2):101-16.

7. Mehmood A, Shah AH, Khan SM, Rehman IU, Ahmad $\mathrm{H}$. Floristic list and indigenous uses of Poaceae family in District Tor Ghar, Khyber Pakhtunkhwa, Pakistan. J Appl Environ Biol Sci. 2017; 7(6):169-77.

8. Piperno D, Sues HD. Dinosaurs dined on grass. Science. 2005; 310(5751):1126-8.

9. Cocucci $A E$, Anton AM. The grass flower: suggestions on its origin and evolution. Flora. 1988;181: 353-62.

10. Ali SI. The significance of flora with special reference to Pakistan. Pak J Bot. 2008; 40:967-71.

11. Chaudhari SK, Arshad M, Ahmed E, Mustafa G, Fatima S, Akhtar $S$, et al. Ethnobotanical evaluation of grasses from Thal Desert, Pakistan. Arc Des Sci. 2013; 66(5):248-55.

12. Samreen U, Ibrar M, Badshah L. Ethnobotanical usages of Poaceae family in district Bannu. Curr Opin Agricult. 2015;4(1):1-4.

13. Anonymous. District Census Report of Charsadda. PCO, Govt. of Pakistan. 1998.

14. Nasir Y, Ali Sl. Flora of Pakistan. PARC, Islamabad, Pakistan. 1970-89.

15. Stewart RR. An annotated catalogue to the vascular plants of west Pakistan and Kashmir. Fakhri Printing Press, Karachi. 1972.

16. Ali SI, Nasir Y. Flora of Pakistan, Karachi and Islamabad, 191-193.

17. Ali SI, Qaiser M. Flora of Pakistan. Department of Botany, University of Karachi. 1993-2018.

18. Raunkiaer $\mathrm{C}$. The life forms of plants and Statistical Plant Geography. Clarendon Press, Oxford. 1934.
19. Hussain F. Field and Laboratory Manual of Plant Ecology. University Grant Commission Islamabad, Pakistan. 1989.

20. Yen $\mathrm{C}$, Yang JL. Historical review and prospect of taxonomy of tribe Triticeae Dumortier (Poaceae). Breed Sci. 2009; 59:513-8.

21. Ahmad F, Khan MA, Ahmad M, Zafar M, Nazir A, Marwat SK. Taxonomic studies of grasses and their indigenous uses in the salt range area of Pakistan. Afr J Bio. 2009; 8(2):231-49.

22. Rafay M, Khan RA, Yaqoob S, Ahmad M. Floristic composition of grass species in the degrading Rangelands of Cholistan Desert. Pak J Agri Sci. 2013; 50(4):599-603.

23. Ahmad F, Khan MA, Ahmad M, Zafar M, Mahmmod $T$, Jabeen $A$, et al. Ethnomedicinal uses of grasses in Salt Range Region of Northern Pakistan. J Med Plant Res. 2010; 4(5):362-9.

24. Ullah Z, Khan MA, Ahmad M, Zafar M, Ullah K. Systematic implications of foliar epidermis in Andropogoneae (Poaceae) from HindukushHimalayas Pakistan. J Med Plant Res. 2011; 5(6):949-57.

25. Khan $M$, Hussain $F$, Musharaf $S$. Floristic Composition and Biological Characteristics of the Vegetation of Sheikh Maltoon Town District Mardan, Pakistan. Annu Res Rev Biol. 2013; 3(1):31-41.

26. Ilyas M, Qureshi R, Shinwari ZK, Arshad M, Mirza SN, Haq ZU. Some ethnoecological aspects of the plants of Qalagai Hills, Kabal Valley, Swat, Pakistan. Int J Agric Biol. 2013; 15(5):801-10.

27. Ali A, Badshah L, Hussain F, Shinwari ZK. Floristic composition and ecological characteristics of plants of Chail Valley, District Swat, Pakistan. Pak J Bot. 2016; 48(3):1013-26.

28. Khan NA, Shah M. Eco-taxonomic study of family Brassicaceae of District Mardan, Khyber PukhtoonKhwa, Pakistan PJLS. 2013; 1(1):28-35.

29. Sher Z, Hussain F, Badshah L, Wahab M. Floristic composition, communities and ecological characteristics of weeds of wheat fields of Lahor, District Swabi, Pakistan. Pak J Bot. 2011; 43(6):281720.

30. Perveen A, QaiserM. Pollen flora of Pakistan-LXIX. Poaceae. Pak J Bot. 2012; 44(2):747-56.

31. Zereen A, Bokhari TZ, Khan ZUD. Ethnobotanical usages of grasses in central Punjab-Pakistan. Int $\mathrm{J}$ Sci \& Eng Res. 2013; 4(9):452-61.

32. Osman A, Zaki Z, Hamed S, Hussein N. Numerical taxonomic study of some tribes of gramineae from Egypt. Am J Plant Sci. 2011; (2):1-14.

33. Gould FW, Shaw RB. "Grass systematics" Second edition Texas A \& M University Press College Station the United States of America. 1983. 